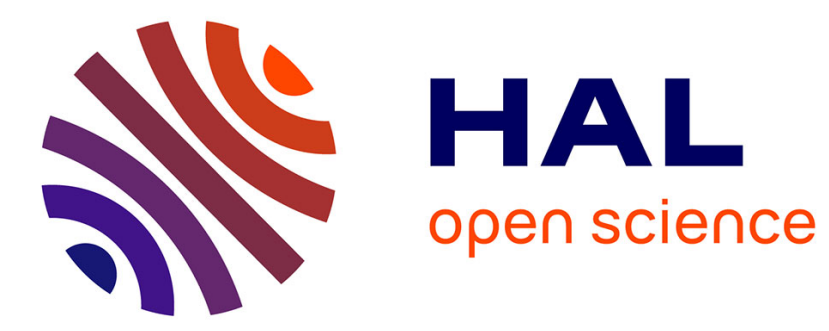

\title{
DAYcast: A Dynamic Transmission Delay Based Broadcast Protocol for Vehicular Ad Hoc Networks
}

$\mathrm{Xu} \mathrm{Wu}$, Jun Zheng, Hui Tong, Nathalie Mitton

\section{To cite this version:}

$\mathrm{Xu}$ Wu, Jun Zheng, Hui Tong, Nathalie Mitton. DAYcast: A Dynamic Transmission Delay Based Broadcast Protocol for Vehicular Ad Hoc Networks. IEEE International Conference on Communications (ICC), Jun 2014, Sydney, Australia. hal-00927712

\section{HAL Id: hal-00927712 \\ https://hal.inria.fr/hal-00927712}

Submitted on 12 Aug 2014

HAL is a multi-disciplinary open access archive for the deposit and dissemination of scientific research documents, whether they are published or not. The documents may come from teaching and research institutions in France or abroad, or from public or private research centers.
L'archive ouverte pluridisciplinaire HAL, est destinée au dépôt et à la diffusion de documents scientifiques de niveau recherche, publiés ou non, émanant des établissements d'enseignement et de recherche français ou étrangers, des laboratoires publics ou privés. 


\title{
DAYcast: A Dynamic Transmission Delay Based Broadcast Protocol for Vehicular Ad Hoc Networks
}

\author{
$\mathrm{Xu} \mathrm{Wu}{ }^{1}$, Jun Zheng ${ }^{1}$, Hui Tong ${ }^{1}$, and Nathalie Mitton ${ }^{2}$ \\ ${ }^{1}$ National Mobile Communications Research Laboratory \\ Southeast University \\ Nanjing, Jiangsu 210096, China \\ ${ }^{2}$ Inria Lille - Nord Europe, \\ 40 Avenue Halley, 59650 Villeneuve D'ASCQ, France \\ Email: junzheng@seu.edu.cn
}

\begin{abstract}
Broadcasting is an effective routing paradigm for data dissemination in vehicular ad hoc networks (VANETs) One concern that arises with broadcasting is the broadcast storm problem, which would cause node contentions and data collisions, and thus degrade the transmission efficiency of a network. This paper proposes a Dynamic trAnsmission delaY based broadcast (DAYcast) protocol for a VANET. To alleviate the effect of the broadcast storm and improve the transmission efficiency of the network, DAYcast only allows the effective neighbors of a source vehicle to broadcast a received data packet and the selection of the effective neighbors are based on the position information on the one-hop neighbors of the source vehicle. Meanwhile, it allows each effective neighbor to wait a certain transmission delay before it broadcasts a received packet. The transmission delay of an effective neighbor depends on the distance between the neighbor and the source vehicle, and the number of effective neighbors of the source vehicle. Simulation results show that DAYcast can effectively improve the network performance in terms of network reachability and the successful delivery ratio as compared with existing weighted p-persistence broadcasting (WPB) and slotted 1-persistence broadcasting (SPB).
\end{abstract}

Keywords-broadcast; broadcast storm; DAYcast; effective neighbor; routing; VANET

\section{INTRODUCTION}

Vehicular ad hoc networks (VANETs) have widely been considered as a promising networking technology for supporting intelligent transportation systems (ITS) [1]. A VANET consists of a number of vehicles moving on highways or roads in an urban area, which may be equipped with sensing devices, radio transceivers, and positioning systems, and can thus provide a variety of applications, such as traffic control, environment monitoring, and inter-vehicle communication [2]. In a VANET, a vehicle or roadside unit (RSU) needs to disseminate relevant information or data to one or multiple destinations via intermediate vehicles or RSUs. For this purpose, a routing protocol is needed to route the information or data to its destinations. Compared with traditional mobile ad hoc networks (MANETs), however, a VANET have some unique characteristics, such as highly dynamic topology, intermittent connectivity, and predictable mobility model, which presents a big challenge for the

This work was supported by the National Natural Science Foundation of China under Grant No. 61372105, the National Science and Technology Major Project under Grant No. 2011ZX03005-004-03, and the Research Fund of National Mobile Communications Research Laboratory, Southeast University, China, under Grant No. 2014A02. design of a routing protocol. Therefore, routing becomes an important issue in the design of a VANET.

Broadcasting is an effective routing strategy for disseminating data in VANETs. In broadcasting, a source node broadcasts a data packet to all its neighbor nodes, which further broadcast the packet until the packet reach its destinations. Compared with unicasting, this routing paradigm has the advantage of simplicity in implementation and can achieve a higher packet delivery ratio. However, broadcasting would introduce the broadcast storm problem which is caused by unlimited or excessive dissemination of the same copy of a data packet in a network. This problem would result in the network to be flooded by a large amount of duplicate packets, which would cause severe node contentions and data collisions, and thus degrade the transmission efficiency of the network. For this reason, it is critical to address the broadcast storm problem in the design of a broadcast protocol in order to improve the transmission efficiency of the network.

In this paper, we propose a Dynamic trAnsmission delaY based broadcast (DAYcast) protocol for data dissemination in a VANET. To alleviate the effect of the broadcast storm and improve the transmission efficiency of the network, DAYcast only allows the effective neighbors of a source vehicle to broadcast a received data packet and the selection of the effective neighbors are based on the position information on the one-hop neighbors of the source vehicle. Meanwhile, it allows each effective neighbor to wait a certain transmission delay before it broadcasts a received packet. The transmission delay of an effective neighbor depends on the distance between the neighbor and the source vehicle, and the number of effective neighbors of the source vehicle. Simulation results were conducted to evaluate the performance of the proposed DAYcast protocol.

This article is organized as follows. Section II reviews related work on broadcast protocols for VANETs. Section II presents the proposed DAYcast protocol. Section IV shows simulation results to evaluate the performance of DAYcast. Section V concludes this paper.

\section{RELATED WORK}

Broadcasting has been widely studied for VANETs and a variety of broadcast protocols have been proposed in the literature [3-11]. In [3], Ma et al. proposed a distributive cross-layer scheme for the design of the control channel in Dedicated Short Range Communication (DSRC) with three 
levels of broadcast services, including cross-layer message priority setting, dynamic receiver-oriented packet repetitions, and farthest relay with distance-based AD timer for multihop broadcast. In [4], Yiannos et al. proposed a novel speed adaptive probabilistic flooding (SAPF) algorithm, which makes a decision to rebroadcast a message based on the speed of a vehicle and relies only on local information. In [5], Korkmaz et al. proposed an urban multi-hop broadcast (UMB) protocol, which only allows the furthest vehicle from the transmitter to rebroadcast the packet by using a blackburst contention approach. In [6], Barradi et al. proposed a new 802.11 based vehicular multi-hop broadcast protocol, called highway multihop broadcast (HMB), which selects the farthest vehicle with the least speed deviation from the source to forward and acknowledge broadcast frames. Moreover, HMB introduces ICTB/FCTB handshake to resolve the hidden node problem and passive forwarder selection to solve the storm problem. In [7], Wisitpongphan et al. quantified the impact of the broadcast storm in terms of message delay and packet loss rate and proposed three probabilistic and timer-based broadcast suppression techniques. However, the solutions proposed in [4-6] attempt to alleviate the broadcast storm from the perspective of MAC layer. The solutions proposed in [7] are only suitable for dense networks and cannot adapt to the density changing scenarios.

In [8], Wisitpongphan et al. developed a statistical traffic model on the data collected to indicate the characteristics of sparse VANETs. In [9], Sou et al. proposed a store-carrybroadcast (SCB) scheme to assist message dissemination by broadcasting over a specific road segment instead of a single vehicle in sparse VANET. An opposite vehicle is used to disseminate the messages to oncoming vehicles traveling on the reverse lane by broadcasting in SCB. In [10], Cho et al. proposed an efficient way to broadcast a safety message to all directions at an intersection with a short delay and without a collision. In [11], Tonguz et al. proposed DVCAST, which relies only on local topology information for handling broadcast messages in VANETs. DV-CAST can operate in all traffic regimes, including sparse and dense scenarios, but is relatively complicated.

\section{DyNAMIC TIMESLOT-BASED BROADCAST PROTOCOL}

In this section, we present the proposed DAYcast protocol for VANETs.

\section{A. Network Model}

We consider a straightway scenario without intersections, where a number of vehicles are moving on the road, which has one or more lanes in each direction, as shown in Fig. 1. The source node can be a vehicle or an RSU. We only consider the vehicles moving in one direction and do not turn to the vehicles moving on the other direction for data forwarding. Moreover, we do not consider car overtaking behavior.

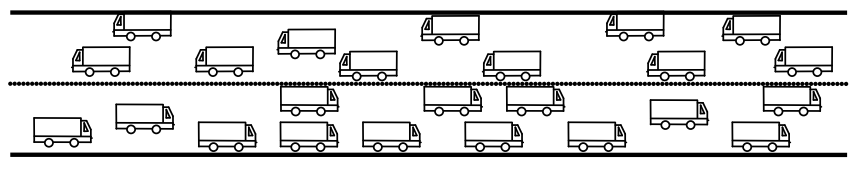

Fig. 1. Network model
We assume that each vehicle is equipped with a GPS device, which can obtain the geographic information on the vehicle. Each vehicle has the same transmission radius. Only when a vehicle is within the transmission radius of another vehicle can the two vehicles communicate with each other.

\section{B. Routing Strategy}

The main idea of the DAYcast protocol is to only allow the effective neighbors of a source vehicle to broadcast a received data packet and allow each intermediate vehicle or node to wait a certain transmission delay before it further broadcasts the received packet in order to reduce node contentions and data collisions, and thus alleviate the effect of the broadcast storm. The selection of the effective neighbors is based on the position information on the onehop neighbors of the source vehicle. The transmission delay of an effective neighbor depends on the distance between the neighbor and the source vehicle, and the number of effective neighbors of the source vehicle. Since the broadcast storm is usually caused by a large number of nodes which simultaneously further broadcast a received packet and contend for a common transmission channel, it is expected to reduce the number of broadcasting nodes and avoid the broadcasting nodes to broadcast data packets simultaneously.

In a VANET, a vehicle is moving at a high speed and its position changes frequently. To support DAYcast, each vehicle needs to obtain its position information (i.e., latitude and longitude) using its GPS device and disseminate the position information to its one-hop neighbors periodically. For this purpose, DAYcast allows a Hello message, which is typically used in a routing protocol, to piggyback the position information in order to reduce the control overhead in the network. According to the received Hello messages, each node that receives a data packet can know the number of its effective one-hop neighbors based on the position information contained in the Hello messages. When a node has a data packet to broadcast, it will first add the number of effective neighbors as well as its own position information (i.e., latitude and longitude) in the data packet, and then broadcast the packet. After a node receives the data packet, it can know the number of its last hop's effective neighbors and determine whether it needs to broadcast and when to broadcast the received packet.

\section{Protocol Description}

The DAYcast protocol consists of two components: effective neighbor selection and dynamic transmission delay calculation.

\section{a) Effective neighbor selection}

The purpose of "effective neighbor" selection for a node that has a data packet to broadcast is to select a group of its one-hop neighbors in the direction of packet transmission as the "effective neighbors" for further broadcasting the packet. To alleviate the broadcast storm, DAYcast does not allow all one-hop neighbors of a source vehicle to further broadcast the packet. Instead, it only allows those one-hop neighbors in the direction of packet transmission to further broadcast the packet, which are called "effective neighbors".

To select the effective neighbors of a node, DAYcast employs a Hello message to exchange position information between different one-hop neighbors of the node. The Hello 
message contains the node ID, and the node's position information (i.e., latitude and longitude), which can be obtained by using the GPS device of the node. After a vehicle receives the Hello messages from all its one-hop neighbors, it extracts the ID, and the latitude and longitude of the source vehicle, compares the source's position with its own position, and determines the number of its effective neighbors.

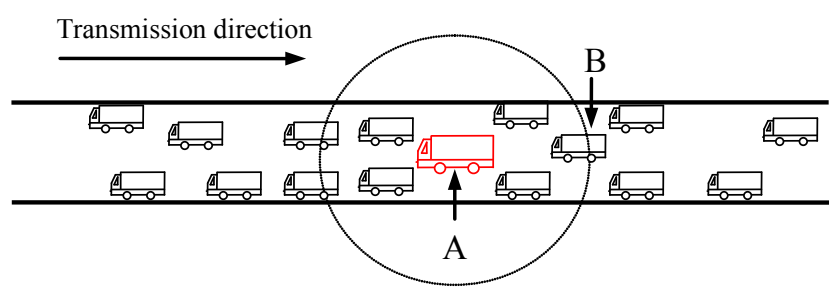

Fig. 2. Illustration of effective neighbor selection

Fig. 2 gives an example for illustrating effective neighbor selection. Assume that a source vehicle A within the dotted circle receives a data packet from its left vehicles and needs to broadcast the packet to its right vehicles. Within its transmission range, vehicle A has seven one-hop neighbors. In this case, the seven neighbor nodes of vehicle A will send Hello messages to vehicle A. But the vehicles to the left of vehicle A have already received the packet and are not in the direction of further transmission relative to vehicle $\mathrm{A}$. Therefore, vehicle A will select the three one-hop vehicles on its right side as its effective neighbors based on the information contained in the Hello messages, i.e., the number of effective neighbors is 3 in this case. In addition to the number of its effective neighbors, vehicle A can also know the maximum distance to its effective neighbors, which is the distance between A and B in this case, based on the Hello messages it has received. When it broadcasts a data packet, it will piggyback its ID, position information, the number of its effective neighbors, and the maximum distance to its effective neighbors.

\section{b) Dynamic transmission delay Calculation}

The purpose of dynamic transmission delay calculation is to dynamically calculate a transmission delay for each effective neighbor to further broadcast its received data packet. Since a source vehicle usually has more than one effective neighbor. To avoid node contentions and data collisions, it is expected that all the effective neighbors do not broadcast their received packet simultaneously. For this purpose, we allow each effective neighbor to broadcast the received packet after a certain transmission delay, which depends on the distance between an effective neighbor and the source vehicle. Obviously, this transmission delay is not equal for different effective neighbors.

Let $N_{j}$ denotes the number of effective neighbors of source vehicle $j, D_{i j}$ is the distance between vehicle $i$ and vehicle $j, D_{\max }$ is the maximum distance between an effective neighbor vehicle and a source vehicle. Thus, the transmission delay for effective neighbor $i$, denoted by $T_{i}$, can be calculated as

$$
T_{i}=\left\llcorner\frac{D_{\max }-D_{i j}}{D_{\max } / N_{j}}\right\lrcorner \times \tau
$$

where

$$
D_{i j}=\sqrt{\left(l a_{i}-l a_{j}\right)^{2}+\left(l o_{i}-l o_{j}\right)^{2}}
$$

and $\left(l a_{i}, l o_{i}\right)$ is the latitude and longitude of vehicle $i$, and $\tau$ is a unit time. Note that $N_{j}, D_{\max }$, and $\left(l a_{i}, l o_{i}\right)$ can be extracted from the received data packet.

\section{c) Protocol procedure}

The major procedure of the proposed DAYcast protocol is given in Fig. 3 and described as follows:

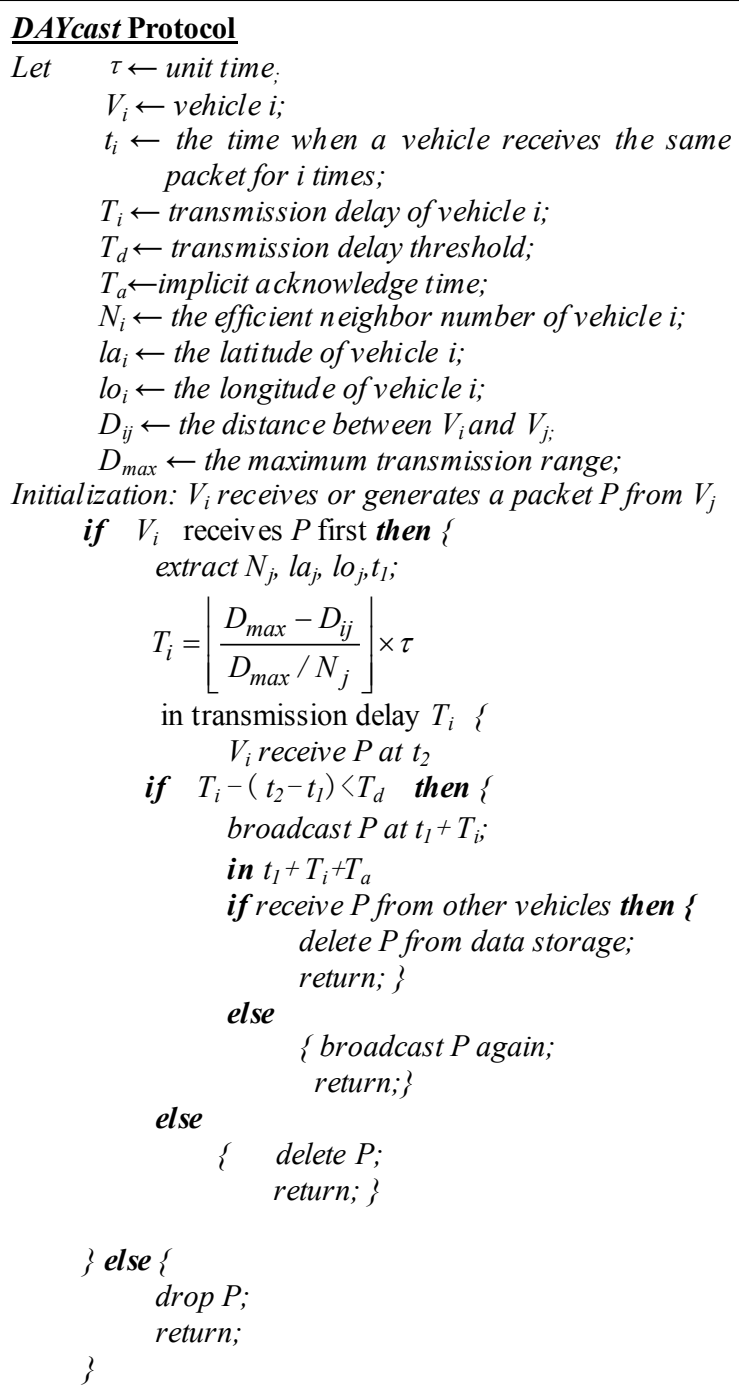

Fig. 3. The procedure of DAYcast protocol

1) When a source vehicle has a data packet to broadcast, it first determines the number of its effective neighbors as well as the maximum distance between an effective neighbor vehicle and the source vehicle based on the information contained in the Hello messages from its one-hop neighbors.

2) Then it adds the number of its effective neighbors, the latitude and longitude of its own, and the maximum distance in the data packet, and broadcast the data to its one-hop neighbors.

3) If a vehicle has received the data packet and broadcast the packet before, it will drop the packet.

4) If a vehicle receives a data packet for the first time, it means that the vehicle is an effective neighbor of the source vehicle. In this case, it will calculate its 
transmission delay for further broadcasting the packet based on Eq. (1). During the transmission delay, if the vehicle receives the data packet again, it will compare the transmission delay with the latest reception time. If the time difference is smaller than a predefined threshold, the vehicle will broadcast the packet at the end of the transmission delay to ensure the network transmission ratio. Otherwise, the vehicle will give up waiting and broadcasting.

5) After a vehicle receives a data packet, it will not explicitly send an acknowledgment message back to the source vehicle. Instead, the source vehicle can know the reception status of its effective neighbors by overhearing the data packet further broadcast by its effective neighbors.

6) If the source vehicle knows that the data packet has already been further broadcasted by its effective neighbors, it will remove the packet from its buffer. Otherwise, it will broadcast the data packet again.

\section{PERFormance EVAluation}

In this section, we evaluate the performance of the DAYcast protocol through simulation results. The simulation experiments are conducted using NS2.35 [12]. In the simulation experiments, we consider a $2 \mathrm{~km}$ straightway scenario with a number of vehicles ranging from 40 to 250 . The source node is a RSU located at the starting end of the straightway. The vehicle driving track is generated using VanetMobiSim [13]. IEEE 802.11 with a transmission rate of $2 \mathrm{Mbps}$ and a transmission range of $250 \mathrm{~m}$ is used as the underlying MAC protocol. The time to live (TTL) of a data packet is set to 100 hops. The running time of each simulation experiment is 500 seconds. All simulation results are averaged over 20 runs. The parameters used in the simulations are summarized in Table I.

TABLE I. SimULATION PARAMETERS

\begin{tabular}{|c|c|}
\hline Parameter & Value \\
\hline Network simulator & NS2 \\
\hline Mobility simulator & VanetMobiSim \\
\hline Simulation area & $2000 \mathrm{~m} \times 10 \mathrm{~m}$ \\
\hline Broadcast interval & $0.05 \mathrm{~s}$ \\
\hline Transmission range & $250 \mathrm{~m}$ \\
\hline Simulation runs & 10 \\
\hline Average vehicle speed & $50 \mathrm{~km} / \mathrm{hr}$ \\
\hline Simulation time & $500 \mathrm{sec}$ \\
\hline Number of vehicles & $40,60,100,150,200,250$ \\
\hline
\end{tabular}

For performance evaluation, we put a virtual node at the $2 \mathrm{~km}$ end of the straightway. We compare the proposed DAYcast protocol with weighted p- persistence broadcasting (WPB) and slotted 1-persistence broadcasting (SPB) proposed in [7]. The reforwarding probability in WPB is supposed to be 0.5 . The performance metrics used in the evaluation are defined as follows:
- Network reachability: The number of data packets received by the virtual node to the total number of data packets broadcast by the source node;

- Successful delivery ratio: The number of vehicles successfully receiving the data packets sent by the source node to the total number of vehicles in the network;

- Average delivery delay: The average time taken for a data packet to be delivered from the source node to the virtual node.

Fig. 4 shows the network reachability with DAYcast, WPB, and SPB under different node density, respectively. It is seen that when the node density is 20 vehicles $/ \mathrm{km}$, the network reachability is very low. This is because in this case the network is not fully connected. With the node density increasing, this performance can reach $80 \%$ when the density is between 30 vehicles $/ \mathrm{km}$ to 75 vehicles $/ \mathrm{km}$. When the density continues to increase, the network will become fully connected and the broadcast storm problem will become more serious. In this case, the network reachability with all the three protocols begins to decline. On the other hand, the network reachability with DAYcast is very close to that with SPB, and both DAYcast and SPB outperform WPB.

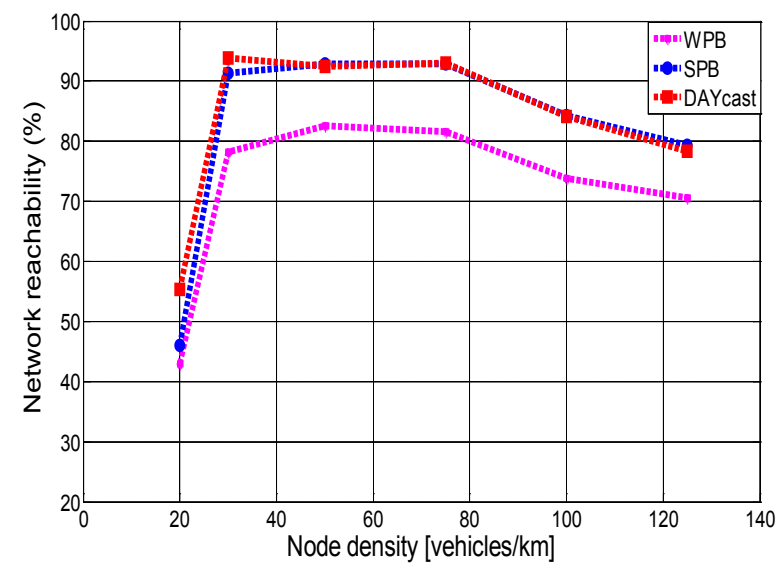

Fig. 4. Network reachability

Fig. 5 shows the successful delivery ratio with DAYcast, SPB, and WPB under different node density, respectively. It is seen that the delivery ratio with DAYcast is $8 \%$ and $15 \%$ larger than that with SPB and WPB, respectively. This is because DAYcast only allows the effective neighbors of a source vehicle to further broadcast a received data packet and meanwhile allows each effective neighbor to wait a different transmission delay before it broadcasts a received data packet, which effectively alleviates the effect of the broadcast storm. On the other hand, when the node density is between 30 nodes $/ \mathrm{km}$ and 75 nodes $/ \mathrm{km}$, the delivery ratios with DAYcast and SPB change slightly. This is because in these cases the number of vehicles in the network is not large and the node contentions and data collisions are not serious. Both DAYcast and SPB can handle the contentions and collisions effectively. For WPB, however, the ratio decreases with the increase of the node density. With the node density further increasing, the delivery ratios with all the three solutions decrease. The reason is that in these cases the broadcast storm problem becomes more serious, which make all the three solutions unable to handle it satisfactorily. 
Fig. 6 shows the average delivery delay with DAYcast, $\mathrm{SPB}$, and WPB under different density, respectively. It is seen that the delay with WPB changes slightly, while that with either DAYcast or SPB increases with the increase of the node density. This is because with either DAYcast or SPB a vehicle needs to wait a certain transmission delay before it broadcasts a received data packet. According to Eq. (1), the larger the node density is, the longer the transmission delay, causing the results.

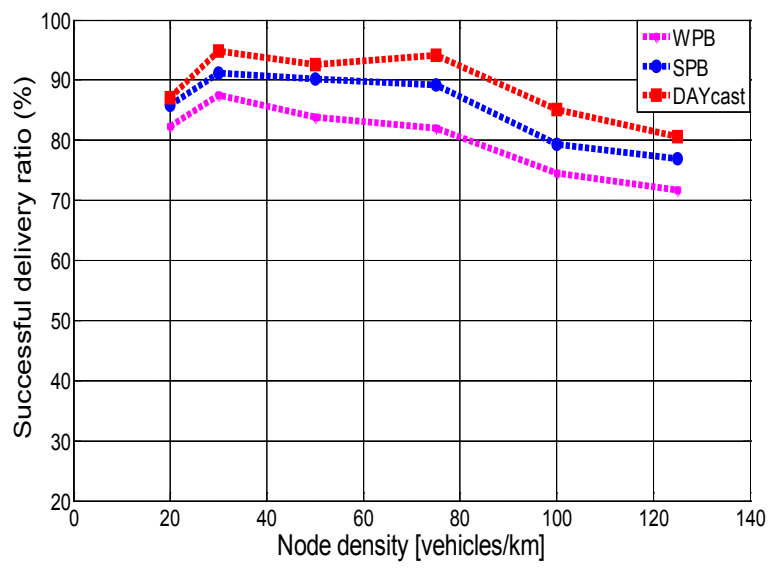

Fig. 5. Successful delivery ratio

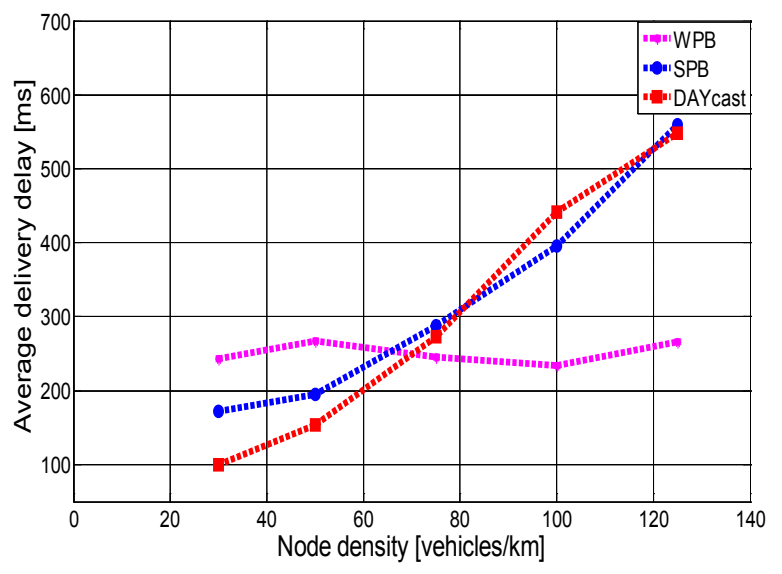

Fig. 6. Average delivery delay

\section{CONCLUSIONS}

In this paper, we proposed a dynamic transmission delay based broadcast (DAYcast) protocol for VANETs. To alleviate the effect of the broadcast storm and improve the transmission efficiency of the network, DAYcast only allows the effective neighbors of a source vehicle to broadcast a received data packet and the selection of the effective neighbors are based on the position information on the onehop neighbors of the source vehicle. Meanwhile, it allows each effective neighbor to wait a certain transmission delay before it broadcasts a received packet. The transmission delay of an effective neighbor depends on the distance between the neighbor and the source vehicle, and the number of effective neighbors of the source vehicle. That is to say, DAYcast chooses the effective neighbors to reduce the number of vehicles which broadcast the received packet, and then calculates the transmission delay to avoid the contention. Thereby, DAYcast eliminates the influence of the broadcast storm to the packet transmission in a VANET. Simulation results show that DAYcast can effectively improve the network performance in terms of network reachability and the successful delivery ratio as compared with existing weighted p-persistence broadcasting (WPB) and slotted 1persistence broadcasting (SPB). In future work, we will consider a more practical road scenario with intersections and study relevant issues for the design of efficient broadcast protocols.

\section{REFERENCES}

[1] R. L. Courtney, "A broad view of ITS standards in the US", in Proc. of the 8th IEEE International Conference on Intelligent Transportation Systems, Washington, DC, Nov. 1997, pp.529-536.

[2] F. Kong and J. Tan, "A collaboration-based hybrid vehicular sensor network architecture", in Proc. of 2008 International Conference on Information and Automation (ICIA'08), 2008, pp. 584-589.

[3] X. Ma, J. Zhang, X. Yin, and K. S. Trivedi, "Design and analysis of a robust broadcast scheme for VANET safety-related services," IEEE Transactions on Vehicular Technology, vol. 61, no.1, Jan. 2012, pp.46-60.

[4] M. Yiannos, L. Marios, and P. Andreas, "Speed adaptive probabilistic flooding in cooperative emergency warning," in Proc. of $4^{\text {th }}$ Annual International Conference on Wireless Internet (WICON'08), Maui, Hawaii, Nov. 2008.

[5] G. Korkmaz et al., "Urban multi-hop broadcast protocol for intervehicle communication systems," in Proc. of ACM Int'l. Wksp. Vehic. Ad Hoc Networks, Philadelphia, PA, Oct. 2004.

[6] M. Barradi, A.S. Hafid, and S. Aljahdali, "Highway multihop broadcast protocols for vehicular netwroks," in Proc. of 2012 IEEE International Conference on Communications (ICC'12), Ottawa, ON, Jun, 2012, pp. 5296-5300.

[7] N. Wisitpongphan, O.K. Tonguz, J.S. Parikh, P. Mudalige, F. Bai, and V. Sadekar, "Broadcast storm mitigation techniques in vehicular ad hoc networks," IEEE Wireless Communications, vol. 14, no. 6, Dec, 2007, pp. 84-94.

[8] N. Wisitpongphan, Bai Fan, P. Mudalige, and O.K. Tonguz, "On the routing problem in disconnected vehicular ad hoc networks," in Proc. of $26^{\text {th }}$ IEEE International Conference on Computer Communications (INFOCOM'07), Anchorage, AK, May, 2007, pp. 2291-2295.

[9] S. Sok-Ian, L. Yinman, "SCB: store-carry-broadcast scheme for message dissemination in sparse VANET," in Proc. of IEEE $75^{\text {th }}$ Vehicular Technology Conference (VTC Spring), Yokohama, May, 2012, pp. 1-5.

[10] C. Jinyoun, S. Seokjoo, and J. Copeland, "Fast broadcast at the intersection in VANET," in Proc. of IEEE Consumer Communications and Networking Conference (CCNC), Las Vegas, NV, Jan, 2011, pp. 65-69.

[11] O. K. Tonguz, N. Wisitpongphan, and B. Fan, "DV-CAST: a distributed vehicular broadcast protocol for vehicular ad hoc networks," IEEE Wireless Communications, vol. 17, no. 2, Apr, 2010, pp. 47-56.

[12] NS-2, available at http://www.isi.edu/nsnam/ns/

[13] J. Harri, F. Filali, C. Bonnet, and M. Fiore, "VanetMobiSim: generating realistic mobility patterns for VANETs," in Proc. of the 3rd International Workshop on Vehicular Ad Hoc Networks, New York, NY, 2006, pp. 96-97. 